\title{
Compact and Wideband Disc Monopole Antenna based on Epsilon Negative Transmission Line for WiFi Applications
}

\author{
Parisa Forouzannezhad \\ Florida International University \\ Miami, Florida
}

\author{
Sahereh SahandAbadi \\ Razi University \\ Kermanshah, Iran
}

\author{
Morteza Azizi Ghoomi \\ Islamic Azad University \\ Tehran, Iran
}

\begin{abstract}
In this work, a compact and broadband and planar monopole antenna consists of one unit cell of epsilon negative transmission line (ENG TL) is proposed. A disc-shaped monopole antenna is implemented at $2.45 \mathrm{GHz}$ resonance frequency for $2.4 \mathrm{GHz}$ applications. A $50 \Omega$ microstrip line is used as a feedline and element of the antenna has $0.1 \lambda_{0}$ of diameter. The size of the antenna is reduced to $0.32 \lambda_{0} \times 0.32 \lambda_{0}$, and the $-10 \mathrm{~dB}$ fractional bandwidth is improved to $12.8 \%$ due to using metamaterial transmission line. Prototype antenna is fabricated and tested, and the measured results are compared to the simulated results using Ansoft HFSS.
\end{abstract}

\section{Keywords}

Compact antenna, Microstrip feedline, Epsilon negative transmission line, ZOR antenna

\section{INTRODUCTION}

Small antenna design is a challenging problem for many researchers. The growing interest in this field originated from the widespread use of modern systems which requires broadband antennas with a small form factor. A small electric antenna can be defined as an antenna which possesses geometrical dimension that is small in comparison to the wavelength of the electromagnetic fields they radiate [1, 2]. These antennas have various applications in unmanned vehicles [3, 4, 5], radars [6], and wireless communication [7, 8] which motivate researchers to develop these antennas based on their needs.

Metamaterial structures which are first developed by V. G. Veselago in 1967 provide electromagnetic properties that do not exist in nature and include negative index of refraction, negative permittivity, or negative permeability [9]. Transmission line based metamaterials that are introduced in [10, 11] provide a conceptual route for implementing small resonant antennas [12]. In addition, one type of the transmission line based metamaterials is ENG-TL that has negative effective permittivity at low-frequency ranges and zero at a special frequency. At this zeroth-order resonance frequency, the frequency does not depend on the length of the antenna but on lumped elements of the ENG TL [13].

One of the most interesting applications of the transmission line based metamaterial is designing and implementation of miniaturized antennas. Many studies in this field have been carried out

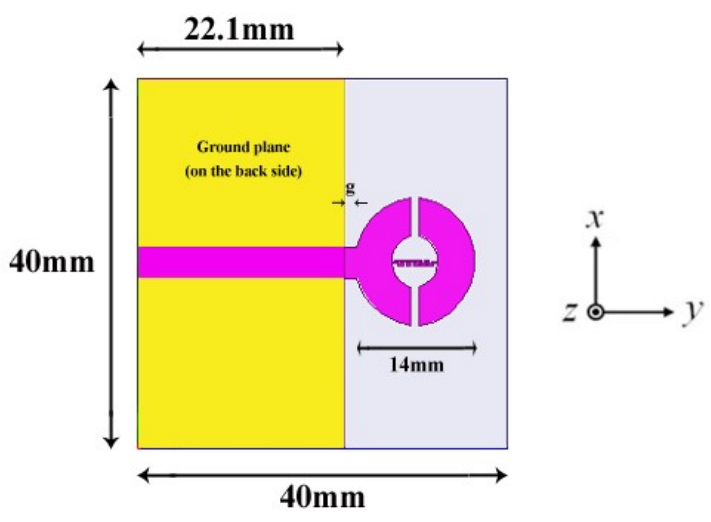

Fig. 1. Configuration of the proposed antenna

that resulted in designing various electrically small antennas using zeroth-order resonator operating at ZOR frequency [14]. However, while small-size antenna design is feasible at this frequency, many of these antennas possess narrow bandwidth that limits their use for some applications. Due to this deficiency, the bandwidth increment at TL-based metamaterial antennas is now defined as one of the leading research goals.

Miniaturization of the antenna has been studied in recent years for different applications [15]. In [16] a very small size antenna with dimensions of $0.07 \lambda_{0} \times 0.04 \lambda_{0}$ at $2.45 \mathrm{GHz}$ for WiFi applications was designed and fabricated that provided $90 \mathrm{MHz}$ of bandwidth. In [17], a circular-shaped monopole antenna which had the same dimensions of $0.07 \lambda_{0} \times 0.04 \lambda_{0}$ was implemented that had 220 $\mathrm{MHz}$ fractional bandwidth at $2.3 \mathrm{GHz}$ resonance frequency.

In this study, a relatively compact $\left(0.1 \lambda_{0}\right.$ of diameter $)$ monopole antenna with extended bandwidth (up to $320 \mathrm{MHz}$ ) at $2.45 \mathrm{GHz}$ of resonant frequency using ENG TL meander lines is proposed for WiFi applications. The simulated return loss and radiation patterns are presented, and the measurement results of the fabricated antenna are compared to the simulations as well.

\section{ANTENNA DESIGN}

The proposed antenna is designed on Rogers/RT duroid 4003 substrate with a thickness of $1.524 \mathrm{~mm}, \epsilon_{r}=3.55$. The antenna is fed 

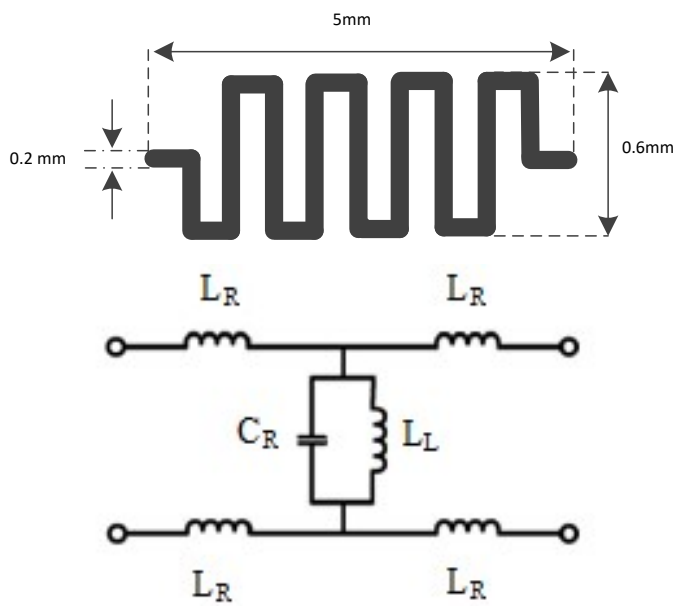

Fig. 2. Unit cell of ENG TL (top) and equivalent circuit model for the unit cell (bottom)

by a $50 \Omega$ microstrip line with a length of $24.1 \mathrm{~mm}$ and width of 3.4 $\mathrm{mm}$. The overall size of the monopole antenna used in this study is $40 \mathrm{~mm} \times 40 \mathrm{~mm}$, and the antenna has the disc shape element with $14 \mathrm{~mm}$ of diameter.

The structure of the proposed antenna and the unit cell with its equivalent circuit model are depicted in Fig. 1 and Fig. 2. The ZOR antenna is an open-ended resonator, thus independent resonant frequency of the physical size can be achieved through a unit cell of epsilon negative meander transmission line. In this design, throughout the current flow on the meander line and the circular patch, the shunt and series inductances can be obtained, and between the two adjacent lines, the shunt capacitance $\left(C_{R}\right)$ has been formed. The various amount of ZOR frequency can be obtained by changing the length and width of unit cell which results in different amounts of $L_{R}$ and $C_{R}$ according to [18]

$$
f_{0}=\frac{1}{2 \pi \sqrt{L_{L} \cdot C_{R}}}
$$

In this proposed model, the meander line has a width of $0.2 \mathrm{~mm}$. The length and width of the unit cell to adjust the resonant frequency at $2.45 \mathrm{GHz}$ for wireless applications are calculated. The length of the unit cell is $5 \mathrm{~mm}$ and the width is $0.6 \mathrm{~mm}$. Based on [19] the quality factor of ZOR antenna is calculated as

$$
Q=\frac{1}{G} \sqrt{\frac{C_{R}}{L_{L}}}
$$

Where $G$ is shunt conductance of the transmission line

\section{SIMULATION AND MESEARMENT RESULTS AND DISCUSSION}

An open-ended resonator antenna has a narrow bandwidth since $Q$ depends on $C_{R}$ and $L_{L}$. On the other hand, the fractional bandwidth is the inverse of the quality factor; increases the amount of $L_{L}$ and decreases the amount of $C_{R}$ results that improved the bandwidth [19]. In this study, the focus is on a large $L_{L}$ and small $C_{R}$ using meander lines which can enhance BW. It should be mentioned that a relatively long microstrip feedline increases the total size of antenna that is important for input impedance
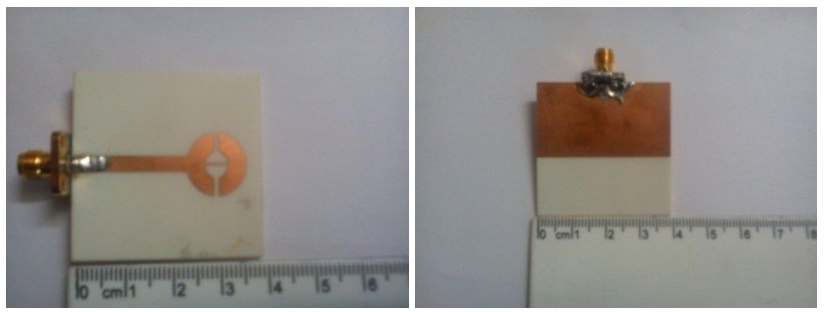

Fig. 3. Top view (Left) and bottom view (Right) of fabricated monopole antenna

matching. The overall size of the antenna can become smaller by using a matching network in substitution of present feedline [18].

The top and bottom views of the fabricated antenna are depicted in Fig 3 Using HFSS software, full-wave simulation results are obtained.

The measured $S_{11}$ parameter compared to the simulated result is shown in Fig. 4 As seen the simulated resonant frequency is 2.45 $\mathrm{GHz}$ with $S_{11}$ at $-18 \mathrm{~dB}$. The $-10 \mathrm{~dB}$ bandwidth is $310 \mathrm{MHz}$ from $2.28 \mathrm{GHz}$ to $2.59 \mathrm{Ghz}$. Compared to [16] and [17] the proposed antenna exhibits more bandwidth of $90 \mathrm{MHz}$ and $220 \mathrm{MHz}$, respectively. The measured resonant frequency is at $2.5 \mathrm{GHz}$ with $S_{11}$ at $-15.5 \mathrm{~dB}$ and $320 \mathrm{MHz}$ of $10 \mathrm{~dB}$ bandwidth from $2.36 \mathrm{GHz}$ to $2.68 \mathrm{GHz}$.

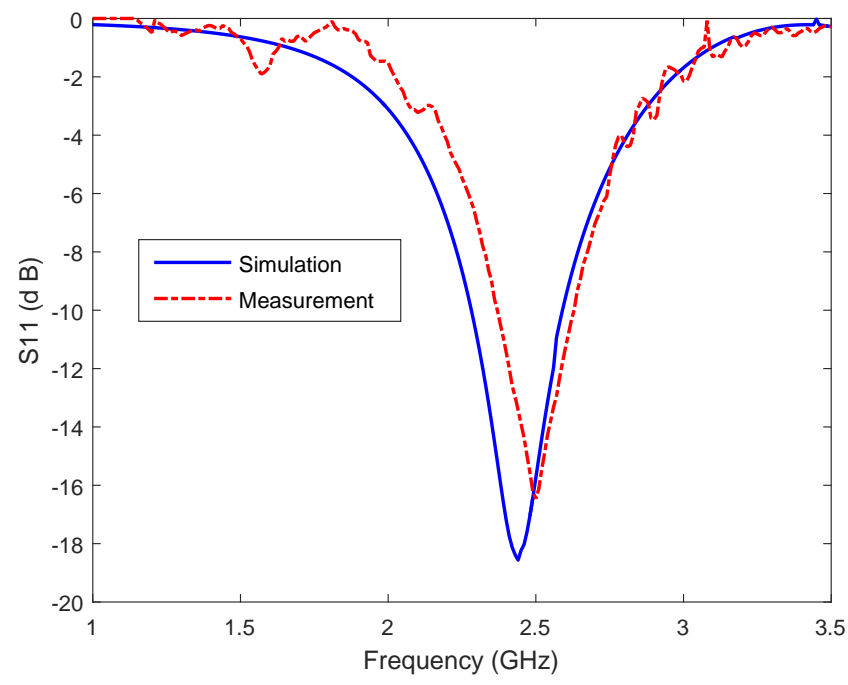

Fig. 4. Simulated and measured return loss of the proposed antenna

Fig. 5 shows HFSS simulated current distribution on the monopole antenna at $2.4 \mathrm{GHz}$. At the center frequency, there is a strong current on the meander line which acts as a radiation element, and thus it is expected that the radiation pattern of the planar antenna in Eplane is in the shape of 8 at $2.45 \mathrm{GHz}$. Also, the H-plane radiation pattern is purely omni-directional at the same frequency.

The simulated 2D radiation pattern of the proposed antenna for different frequencies from $1.5 \mathrm{GHz}$ to $3.5 \mathrm{GHz}$ were studied. As predicted, the antenna has approximately a donut-like radiation pattern similar to that short monopole over a small ground plane. It was observed that as the frequency moves toward the upper levels, the radiation pattern is somehow slightly distorted (for fre- 


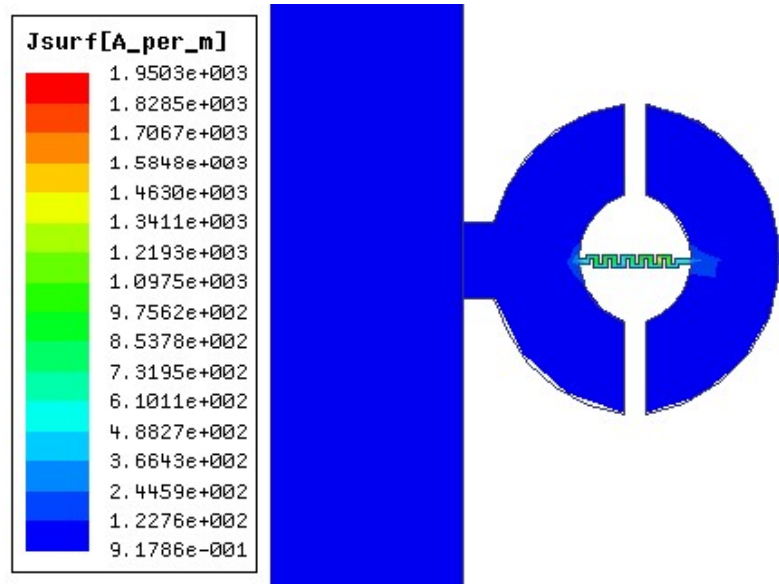

Fig. 5. Simulated current distribution of antenna at $2.4 \mathrm{GH}$

quencies higher than $3.5 \mathrm{GHz}$ ). The transition of the pattern from a dipole-like at the lower frequencies to the slowly distorted pattern at the higher frequencies indicates that this antenna must have experienced great variations in its behavior, however, it preserved omni- directionality all over the frequency band from $1.5 \mathrm{GHz}$ to $3.5 \mathrm{GHz}$. This is due to the small ground plane, the small gap between the ground plane and the patch, ' $\mathrm{g}=2 \mathrm{~mm}$ ', which is a leading factor for good impedance matching of the antenna and appropriate reflection coefficient as a result. In summary, it is obvious that the $2.4 \mathrm{GHz}$ planar monopole antenna almost possesses constant radiation patterns and linear electric field polarization throughout the frequency bandwidth.

In Fig. 6 and 7. measured radiation patterns at $2.4 \mathrm{GHz}$ for copolarization and cross-polarization in H-plane and E-plane compared to the simulated patterns. As it is obvious, the measured results are in agreement with simulation but slightly distorted due to inaccuracy at the test equipment and fabrication limitations.

The input impedance $\left(Z_{i n}=R+j X\right)$ of the antenna at the resonant frequency has the $48 \Omega$ of the real part and the $-12 j \Omega$ of the imaginary component which shows a relatively good impedance matching. Moreover, $1.8 \mathrm{dBi}$ of the HFSS-simulated gain for the proposed antenna at operating frequency is obtained.

According to the equivalent circuit model of unit cell shown in Fig 2 and using HFSS and ADS, $L_{R}$ is equal to $0.85 \mathrm{nH}$, also $C_{R}=1.11 \mathrm{pF}$, and $L_{L}=28.4 \mathrm{nH}$ are obtained that leads to 42.81 $\mathrm{GHz}$ of zeroth-order resonant frequency. The size of the groundplane plays a significant role in the performance of the antenna. In order to investigate how the ground-plane size affect the antenna performance, the simulation has been run for different ground length (in direction of y-axis), and the results are presented in Table 1 . As seen the gain and bandwidth are slightly increase with increasing the ground width, and a small decrement in resonant frequency is occured. In Table 2, the proposed antenna performance is compared to some of the previous compact antennas which work at $2.4 \mathrm{GHz}$. It is clear that the proposed antenna is small and wideband in comparison with the similar antennas.

\section{CONCLUSION}

A planar monopole disc-shaped antenna based on metamaterial is proposed which is compact in size by loading a unit cell of epsilon negative transmission line. The overall size of antenna is $0.32 \lambda_{0} \times 0.32 \lambda_{0}$ and the diameter of element is $0.1 \lambda_{0}$ with dipole-

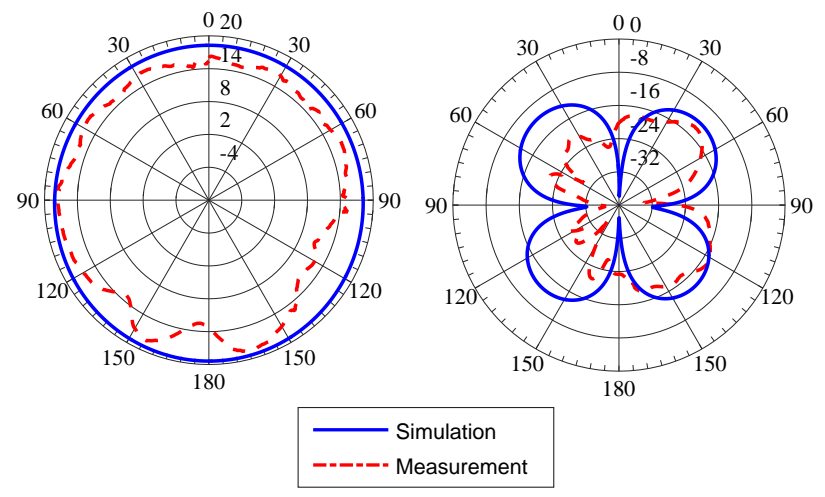

Fig. 6. Simulated and measured radiation patterns at $2.4 \mathrm{GHz}$ in $\mathrm{H}$-plane for co-polarization (Left) and cross-polarization (Right)

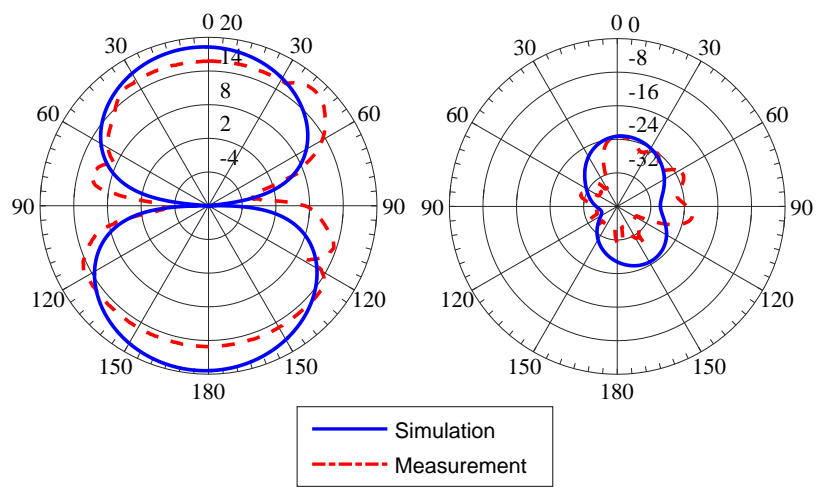

Fig. 7. Simulated and measured radiation patterns at $2.4 \mathrm{GHz}$ in E-plane for co-polarization (Left) and cross-polarization (Right)

like radiation patterns. In addition, the antenna has $1.8 \mathrm{dBi}$ of gain and $-10 \mathrm{~dB}$ bandwidth of $320 \mathrm{MHz}$ illustrates extended bandwidth. The monopole antenna can be easily matched due to the gap between the patch and feedline. The proposed model is fabricated and tested. The measured return loss and patterns and the simulated results are in agreement. In comparison to the previous studies, this structure is compact, broadband around $2.4 \mathrm{GHz}$ for WiFi applications and it is easy to fabricate. 


\section{REFERENCES}

[1] J. S. McLean, "A re-examination of the fundamental limits on the radiation q of electrically small antennas," IEEE Transactions on antennas and propagation, vol. 44, no. 5, p. 672, 1996.

[2] D. F. Sievenpiper, D. C. Dawson, M. M. Jacob, T. Kanar, S. Kim, J. Long, and R. G. Quarfoth, "Experimental validation of performance limits and design guidelines for small antennas," IEEE Transactions on Antennas and Propagation, vol. 60, no. 1, pp. 8-19, 2012.

[3] A. Abaspour, S. H. Sadati, and M. Sadeghi, "Nonlinear optimized adaptive trajectory control of helicopter," Control Theory and Technology, vol. 13, no. 4, pp. 297-310, 2015.

[4] A. Abbaspour, K. K. Yen, S. Noei, and A. Sargolzaei, "Detection of fault data injection attack on uav using adaptive neural network," Procedia computer science, vol. 95, pp. 193-200, 2016.

[5] A. Abbaspour, P. Aboutalebi, K. K. Yen, and A. Sargolzaei, "Neural adaptive observer-based sensor and actuator fault detection in nonlinear systems: Application in uav," ISA transactions, vol. 67, pp. 317-329, 2017.

[6] D. McLaughlin, D. Pepyne, B. Philips, J. Kurose, M. Zink, D. Westbrook, E. Lyons, E. Knapp, A. Hopf, A. Defonzo, et al., "Short-wavelength technology and the potential for distributed networks of small radar systems," Bulletin of the American Meteorological Society, vol. 90, no. 12, pp. 17971817, 2009.

[7] M. Mafi, "Integration of mobile ad hoc and wimax networks with approach of admission control and hand off combination applied in telemedicine services," American Journal of Scientific Research, vol. 83, pp. 14-24, 2012.

[8] M. Mafi, H. Azizi, and H. Y. Alborz, "A new model of free global positioning system using triple dme," 2017.

[9] V. G. Veselago, "The electrodynamics of substances with simultaneously negative values of and $\mu$," Soviet physics uspekhi, vol. 10, no. 4, p. 509, 1968.

[10] A. A. Oliner, "A planar negative-refractive-index medium without resonant elements," in Microwave Symposium Digest, 2003 IEEE MTT-S International, vol. 1, pp. 191-194, IEEE, 2003.

[11] C. Caloz and T. Itoh, "Application of the transmission line theory of left-handed (lh) materials to the realization of a microstrip" lh line"," in Antennas and Propagation Society International Symposium, 2002. IEEE, vol. 2, pp. 412-415, IEEE, 2002.

[12] J. Zhu and G. V. Eleftheriades, "A compact transmission-line metamaterial antenna with extended bandwidth," IEEE antennas and wireless propagation letters, vol. 8, pp. 295-298, 2009.

[13] A. Lai, K. M. Leong, and T. Itoh, "Infinite wavelength resonant antennas with monopolar radiation pattern based on periodic structures," IEEE Transactions on Antennas and Propagation, vol. 55, no. 3, pp. 868-876, 2007.

[14] J.-Q. Huang and Q.-X. Chu, "Compact epsilon negative zeroth-order resonator antenna with higher radiation efficiency," Microwave and Optical Technology Letters, vol. 53, no. 4, pp. 897-900, 2011.

[15] P. Forouzannezhad, A. Jafargholi, and A. Jahanbakhshi, "Multiband compact antenna for near-field and far-field rfid and wireless portable applications," IET Microwaves, Antennas \& Propagation, vol. 11, no. 4, pp. 535-541, 2016.

[16] J. Zhu and G. Eleftheriades, "Dual-band metamaterialinspired small monopole antenna for wifi applications," Electronics Letters, vol. 45, no. 22, pp. 1104-1106, 2009.

[17] Y. He and G. V. Eleftheriades, "Metamaterial-inpsired wideband circular monopole antenna," in Antennas and Propagation Society International Symposium (APSURSI), 2012 IEEE, pp. 1-2, IEEE, 2012.

[18] M. S. Majedi and A. R. Attari, "A compact and broadband metamaterial-inspired antenna," IEEE Antennas and Wireless Propagation Letters, vol. 12, pp. 345-348, 2013.

[19] T. Jang, J. Choi, and S. Lim, "Compact coplanar waveguide (cpw)-fed zeroth-order resonant antennas with extended bandwidth and high efficiency on vialess single layer," IEEE Transactions on Antennas and Propagation, vol. 59, no. 2, pp. 363-372, 2011.

[20] X. Quan, R. Li, Y. Cui, and M. M. Tentzeris, "Analysis and design of a compact dual-band directional antenna," IEEE Antennas and Wireless Propagation Letters, vol. 11, pp. 547550, 2012.

[21] Y.-S. Wang, M.-F. Hsu, and S.-J. Chung, "A compact slot antenna utilizing a right/left-handed transmission line feed," IEEE Transactions on Antennas and Propagation, vol. 56, no. 3, pp. 675-683, 2008. 
Table 1. Simulated Characteristics of Antenna for Different Sizes of Ground plane

\begin{tabular}{|c|c|c|c|}
\hline Size of the ground plane $(m m \times m m)$ & Resonance frequency $(\mathrm{GHz})$ & $-10 \mathrm{~dB}$ bandwidth $(\mathrm{MHz})$ & Gain $(\mathrm{dBi})$ \\
\hline $22.1 \times 20$ & 2.53 & 270 & 1.70 \\
\hline $22.1 \times 30$ & 2.49 & 300 & 1.74 \\
\hline $22.1 \times 30$ & 2.45 & 310 & 1.80 \\
\hline
\end{tabular}

Table 2. Comparison Results of the Proposed Antenna and Some References

\begin{tabular}{|l|l|l|l|}
\hline References & Element size of the antenna & Overall size of the antenna & $-10 \mathrm{~dB}$ Bandwidth $(\mathrm{MHz})$ \\
\hline Proposed antenna & $0.1 \lambda_{0}$ (diameter) & $0.32 \lambda_{0} \times 0.32 \lambda_{0}$ & 320 \\
\hline$[16]$ & $0.07 \lambda_{0} \times 0.04 \lambda_{0}$ & $0.26 \lambda_{0} \times 0.19 \lambda_{0}$ & 90 \\
\hline$[20]$ & $0.20 \lambda_{0} \times 0.34 \lambda_{0}$ & $0.48 \lambda_{0} \times 0.80 \lambda_{0}$ & 120 \\
\hline$[21]$ & $0.09 \lambda_{0} \times 0.09 \lambda_{0}$ & $0.32 \lambda_{0} \times 0.24 \lambda_{0}$ & 110 \\
\hline
\end{tabular}

